\title{
A Statistical-based Approach to Evaluate the Production of Crawler-type Dozer in Construction Projects
}

\author{
Hamed Nabizadeh Rafsanjani, ${ }^{1, *}$, Yaghob Gholipour ${ }^{2}$, Xiaoxiang Xue ${ }^{3}$ \\ ${ }^{1}$ Durham School of Architectural Engineering and Construction, University of Nebraska-Lincoln, Lincoln, NE 68588, USA; \\ formerly, School of Civil Engineering, University of Tehran, Tehran 14155, Iran \\ ${ }^{2}$ School of Civil Engineering, University of Tehran, Tehran 14155, Iran \\ ${ }^{3}$ Durham School of Architectural Engineering and Construction, University of Nebraska-Lincoln, Omaha, NE 68182, USA; Project \\ Engineer, Jacobs Engineering Group, Omaha, NE 68134, USA. \\ *Corresponding author: hnabizadehrafsanj2@unl.edu
}

\begin{abstract}
Estimating the actual production rate of construction machinery which clearly differs from nominal production provided by machinery manufacturers always is a critical challenge in construction projects execution. Studies indicate that true estimation of actual production is a key element in estimating the time and cost required to terminate construction operations. However, current literature shows that it is still a quite challenging to estimate actual production. In particular, there are various independent parameters that affect the actual production rate. Understanding the role and importance of each parameter could lead to an accurate estimation for production rates. To this end, this paper presents a statistical-based approach to find the discrepancies between the nominal and actual production rates of crawler-type dozers and to understand how various parameters could affects the actual production rates. The data for the actual production of machine were records from productivity measurement of 39 dozers. Working condition, type of materials, and ground slope are three main independent parameters considered and evaluated for each machine. The results obtained from statistical analyses on the data and a comparison between these results with the data provided by Caterpillar and Komatsu manufacturers show a) the discrepancies between the actual and nominal hourly production, b) the effect of individual parameters on actual production, and c) the relationship between machinery working age and discrepancies. The findings of this study could be a unique help for project managers in planning of machinery and equipment in a project site.
\end{abstract}

Keywords: heavy construction machinery, crawler-type dozer, actual hourly production estimation, production parameters, statistical analysis, case study

Cite This Article: Hamed Nabizadeh Rafsanjani, Yaghob Gholipour, and Xiaoxiang Xue, "A Statisticalbased Approach to Evaluate the Production of Crawler-type Dozer in Construction Projects." American Journal of Civil Engineering and Architecture, vol. 6, no. 2 (2018): 80-92. doi: 10.12691/ajcea-6-2-5.

\section{Introduction and Motivation}

Construction machinery and equipment are one of the necessary resources to the accomplishment and success of construction projects. Today contractors undertake many types of construction activities that require different types and sizes of machinery and equipment [1], and they typically invest the largest value of project costs in machinery and equipment specifically for the equipment-intensive projects $[2,3]$. In this context, adequate knowledge of machinery and equipment management critically needs to decrease various risks associated with increasing the overall project cost. Fan et al. [4] and Tatari and Skibniewski [5] provided the critical factors for machinery management. In particular, the success of a construction project is highly connected to its machinery production, and it has been universally accepted that the machinery hourly production is the key factor in success of construction projects [1,3,6-12]. This factor is the main relationship between the machinery management and construction project performance [3]. Machinery manufacturers generally provide an ideal hourly production of their machinery for users. This ideal hourly production named nominal hourly production clearly differs from the actual hourly production of a machine in construction projects. The actual production depends mainly on the conditions of project sites. Estimating actual production and therefore the discrepancies between the nominal and actual production rate is a key element in estimating the time and cost required to terminate the construction operations. In order to estimate this production, it is a critically need to know how different project site conditions affect the machinery production [3]. In fact, since the ideal conditions considered by manufacturers could rarely be achieved in real construction projects, the actual production could differ with the nominal production.

A growing body of research has studied different subjects of construction machinery and equipment management [13-21]. Within this area of interest, researchers have extensively provided new algorithms, methods, models, and techniques to study about 
acquisition, costing, maintenance, purchase, repair, replacement, retirement, selection, and sustainability topics [2,5,22]. In addition, various related computer software has been developed and proposed to help project managers make decisions about construction machinery. In particular, the success of such research work and software is highly depends on machinery actual production [9,11,23-26]. Rafsanjani et al. [10] discussed that the nominal to actual hourly production could get a ratio of less than 0.3 in a project site. Such a high efficiency drop might critically affect results and conclusions by machinery research working on other fields (e.g., costing, replacement, and selection). However, current literature shows that the accurate estimation of hourly production has only intrigued very little research in the past decades. In 1994, Edmonds et al. [27] took the actual production of various earthmoving operation machinery into account, and viewed the actual production as a percentage of full capacity. By using several methods such as short range analysis and analysis of running timespeed, they finally conclude that the actual production of machinery is approximately 52.5 percent of the nominal production. It is worth mentioning that providing a specific amount of production drop for a range of machinery is not a feasible approach being implemented in different project sites, and therefore their percentage result could not be extended to construction machinery industry. Zou [28] used the 3-D HSV color-space digital image processing method to study how different site conditions could influence on excavator idle time and truck load cycle time. Through the proposed method, he tried to achieve more realistic results of machinery production and discussed how this method can be extended to understand the effects of site conditions on production of other machinery and equipment. However, finally no data for actual hourly production of the machines was provided. Rafsanjani et al. $[8,10]$ studied the hourly production of different machinery models and provided the actual hourly production for some machines: excavators, graders, loaders, and rollers. In their work to find the actual production a three stage analysis was employed: long-range analysis, ratio analysis, and analysis of variance. In addition, the authors provided the actual conditions of construction sites where the data of machines was collected. However, they did not discussed how different projects site conditions affected the machinery production. Based on their previous studies, Rafsanjani et al. [11] then proposed a regression-based model to estimate actual production of wheel-type loader. The model uses the engine power (hp) of machine to estimate the actual production. However, the authors did not discussed how the model could be used for a variety of project conditions. In fact, the model could not be extended in-practice to all models of machines working in various project sites. Recently, Rashidi et al. [23] used a linear mixed model to estimate productivity of a model of dozer. They presented factors affect machine productions. However, their results could not be generalized to different models of dozer typically used in project sites.

In particular, the review of literature apparently shows that over the past 20 years, construction literatures have written little information to advance practical basis for actual machinery production estimation. The fact that there are so few publications about production estimation gives evidence to the fact that less attention has been paid to this domain than to the other main domains of machinery; the available research in production estimation domain is significantly insufficient and not proportional to the importance of the topic. Therefore, in order to advance the basis of topic, this paper considers a case study of various models of crawler-type dozer to evaluate the machine production in project sites and to discuss about the main parameters affect the production.

This paper is organized as follows: research objectives is presented in section 2. Section 3 describes the research methodology. In section 4, the results are provided. Section 5 makes a discussion on the results. Limitations of this research and recommendation for future research work are presented in section 6. Finally, section 7 presents conclusions.

\section{Objectives}

The main objective of this paper is to develop a stepby-step approach in order to assess actual hourly production of crawler type dozer. This is achieved through statistical analyzing the real data of a case study for various models of crawler-type dozer. This objective is translated into three sub-objectives:

(1) Find the discrepancies between the actual and nominal hourly production of the machines. The results of this objective give an overview of how the efficiency of machinery and equipment in a real project site can be found. These results could greatly help estimate the most realistic time machinery and equipment fleet needed to complete their assigned tasks.

(2) Determine the individual parameters which affect the machine production. This step is essential to present why the lowest discrepancies might be achieved.

(3) Determine how a machine working age affects its actual hourly production. A special emphasis on this sub-objective helps to understand the relationship between the working age and hourly production, and how the production can be predicted based on the age.

Addressing these sub-objectives constitutes a significant contribution to the field of machinery management. It is worth mentioning that although this paper only focuses on crawler-type dozer, the approach is inclusive and can be extended to other machinery and equipment employed in construction sites.

The main reason for the selection of dozer for this study is that this machine either crawler or wheel-type is typically the starter machine in a fleet of machinery working in a project site, and it actually acts as a feeder for the machinery fleet. Therefore, any delay in the dozer work could have negatively effects on the other machinery and equipment and thus a negative effect on the project. Therefore, there is a need to know how this machine works in a site. In particular, a crawler-type dozer can work on a variety of soils. While sharp-edged pieces of stone are always a problem for wheel dozer, such pieces are not destructive to a crawler type one [3]. A crawler dozer can also work on mud-slick surface. In addition, compared to other machines and equipment, this machine 
provides the highest power, traction, and flotation needed for doing different variety of working conditions; it can easily operate on a slope of $100 \%$ [3]. Therefore, the crawler-type dozer is often used in project site. It is worth mentioning that Rashidi et al. [23] and Ok and Sinha [26] studied dozers to provide their proposed estimation models. To this end, the focus of this study is on the crawler-type dozer machine.

\section{Methodology}

A step-to-step methodology was developed to achieve the sub-objectives. First, in order to get the actual production data, the author employed a real survey on different models of crawler-type dozer in real projects. Then, manufacturers' publications were used to collect data for the nominal production. Finally, required statistical analyses were employed to analyze all data in order to achieve the sub-objectives.

\subsection{Case Study of Real Projects}

Crawler-type dozer generally used for dozing and making compacted soil, materials, and earth loose in project sites. In construction industry, this machine is mainly used in borrow pits to dig the earth. Therefore, the projects chosen for this case study are five different borrow pits which are located around Iran. These pits have generally been dug for use in different construction projects, e.g. highways and dams. The borrow pits are located in areas containing almost similar climate conditions and annual rainfall. In addition, the local weather of these areas is hot and dry. The soil samples taken from all five borrow pits had very little natural moisture. Therefore, it was assumed the pits soil is dry.

Generally, although bias can occurs at any step of research, data collected during an experiment or survey study is more likely to be biased $[29,30]$. Bias in data could mislead the statistical analysis results and study conclusions. Accordingly, machinery data collected only based on a project could be biased due to certain condition of the project. In this context, five different pits chosen for this study could help avoid the bias in data.

\subsection{Machine Selection}

The choice of machine in this study was limited to two manufacturers' models: Caterpillar, and Komatsu. Caterpillar believes to control more than half of the U.S. construction equipment market and one third of the world market [31]. Komatsu dozers are also typically employed in many earthmoving operation [23]. The machine models considered for this study are as follow:

- Caterpillar: D6N, D6T, D7R, D9T, D10T

- Komatsu: D155A-2, D155A-6, D275A, D375A

Table 1 shows the number and age of each model. There were totally 39 machines with average age of 7.3 working years. In addition, the Caterpillar models worked 6 years on average more than the Komatsu models. The variety range for models and their age could provide this opportunity to understand how the age might affect the productivity of machines. In addition, they can also help prevent bias in data that could happen by only considering a model of machine. It is worth mentioning that while crawler-type dozer could work with a variety of blade such as straight or angel blade, all the machines considered for this research worked with Semi-Universal (SU) blades.

Table 1. Number and Age for each Model

\begin{tabular}{|c|c|c|c|c|c|c|c|c|}
\hline & \multirow{2}{*}{$\frac{\text { Model }}{\text { D6N (H) }}$} & \multirow{2}{*}{$\frac{\text { Number }}{6}$} & \multicolumn{6}{|c|}{ Age (years) } \\
\hline \multirow{5}{*}{ 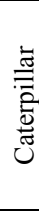 } & & & 8 & 10 & 10 & 11 & 13 & 14 \\
\hline & D6T (G) & 5 & 8 & 9 & 11 & 11 & 14 & \\
\hline & D7R (F) & 6 & 6 & 10 & 10 & 10 & 12 & 13 \\
\hline & D9T (C) & 2 & 4 & 11 & & & & \\
\hline & D10T (B) & 2 & 7 & 9 & & & & \\
\hline \multirow{4}{*}{ 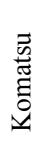 } & D155A-2 & 6 & 2 & 4 & 4 & 5 & 5 & 6 \\
\hline & D155A-6 & 5 & 2 & 2 & 3 & 7 & 7 & \\
\hline & D275A & 3 & 2 & 3 & 5 & & & \\
\hline & D375A & 4 & 3 & 3 & 4 & 6 & & \\
\hline
\end{tabular}

\subsection{Data Collection}

\subsubsection{Nominal Production}

The data for the nominal hourly production was derived from machinery charts and performance handbooks of the two manufacturers: Caterpillar Performance Handbook [32], and Komatsu Specifications \& Application Handbook [33]. Figure 1 presents charts for nominal hourly production of crawler-type dozer. The horizontal axis of each chart shows the value for average of dozing distance while the vertical axis presents the hourly production for different models. The average dozing distance for all models of Komatsu is considered from $15 \mathrm{~m}$ to $180 \mathrm{~m}$. However, the upper bound of this distance is limited to $90 \mathrm{~m}$ for model F, G, and H of Caterpillar, while that of the other models of caterpillar is more than $180 \mathrm{~m}$. In addition, the charts show that the highest hourly production in the ideal case is approximately $2700 \mathrm{Lm}^{3}$ for both Caterpillar and Komatsu dozers.

\subsubsection{Actual Production}

The actual production data was collected from records of different models of dozers in the five borrow pits. In this step, each model was individually considered at a project site. In data gathering phase, based on manufacturers' production charts (see Figure 1) the average dozing distance of machine was considered as a parameter to achieve the production presented in a way that the manufacturers present their machinery production. In this context, the hourly production for all machines in some specified dozing distances was recorded. As shown in Figure 1, the manufacturers typically consider some dozing distances of interest to present their results of hourly production (e.g., $15 \mathrm{~m}, 30 \mathrm{~m}$, and $45 \mathrm{~m}$ ). In the case of this research, before the data collection step, a presurvey was done to determine the typical dozing distance in five pits. Based on the results of this survey, $15 \mathrm{~m}, 30 \mathrm{~m}$, and $75 \mathrm{~m}$ were finally chosen as the three main dozing distance of interest for all models. In other words, these three distances were the only distances that the author collected the real data of all the models. In particular, a comparison between the collected actual data and found nominal data could address the first sub-objective. 
ESTIMATED DOZING PRODUCTION $\bullet$ Semi-Universal Blades $\bullet$ D6N through D11T

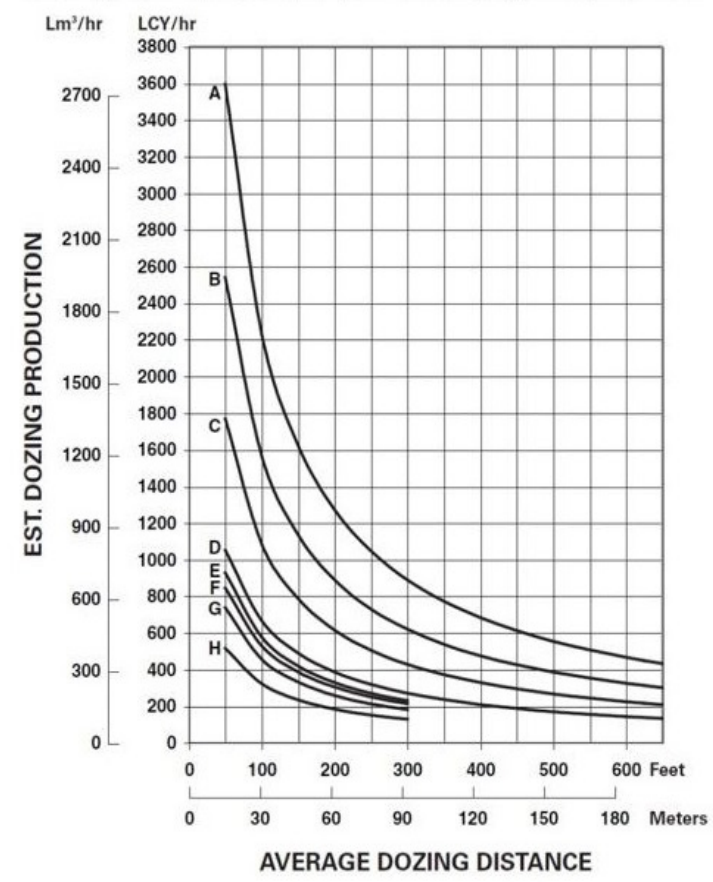

CATERPILLAR
Estimated Dozing Production (Semi-U-tiltdozer)

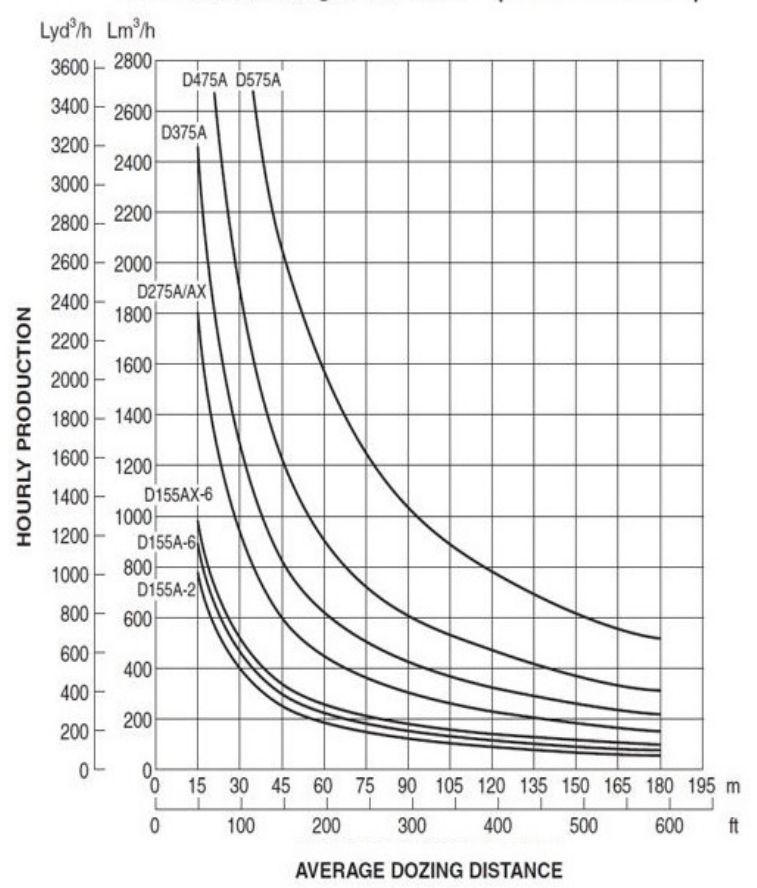

KOMATSU

Figure 1. Manufacturers charts for crawler-type dozer hourly production (Caterpillar performance handbook 2012, Komatsu specifications \& application handbook 2009)

In addition to the dozing distance, working condition, ground slope (i.e., grade\%), and materials (i.e., soil) conditions have critical effects on dozer production [3,10,21,22,23,34]. Indeed, these three independent parameters are the main parameters critically play important roles to estimate dozer actual hourly production. Therefore, in this study, several surveys were conducted on different conditions to study the effect of each parameter on machine productions. In order to address this step as the second sub-objective of this study, it was needed to study various operating conditions of dozer at the borrow pits. In this context, based on the projects conditions, project machinery managers' experience, and the aforementioned literatures, the author categorized each of these three independent parameters as follow:

- Working condition: (1) Good; (2) Medium; (3) Weak

- Ground slope: (1) Zero; (2) $+15 \%$; (3) $-15 \%$

- Types of Materials: (1) Loose soil; (2) Soil containing rubble stones; (3) Blaster rocks

In a good working condition, it was assumed an experienced operator operates a machine with low-working age in an ideal site condition. In this condition, the efficiency for time-working of a machine is approximately $50 \mathrm{~min}$ per hour $[3,9,10,12,32,33]$. 40min per hour and $30 \mathrm{~min}$ per hour are the time-working for medium and weak working conditions, respectively. Depending on various operating terms and conditions, a project site condition can be considered as one of the three working conditions. It must be noticed that the experience of machinery manager in construction projects plays the main role to make this critical decision. In particular, the machinery and equipment manager of five borrow pits has 28 years of experience in earthmoving operations.
Furthermore, the skills and working experience of machine operators also have great influence on machine production. Elazouni and Basha [35] and Alwi [36] mentioned that the poor performance of machine operators can lead to a low production. Figure 2 shows the distribution of working experience of machine operators in the case of this research. The majority of operators were more than five years of working experience. It is noteworthy that the operators assigned randomly to different machines that not only leads to achieve more reliable results extended to machinery industry, but also helps avoid bias in data.

The time of data gathering in the five projects was approximately 8 months. During this period, the dozers run for one 8-hour shift per day and 20 days per month. In particular, it was tried hard to collect the actual production data of each model for various project site conditions during this 8-month period of time that could lead to achieve more reliable results. In addition, a dozer actual hourly production was estimated by dividing the total volume of soil loosed by machine by the total operational hours for a working day.

In addition, based on the three sub-categories considered for each main parameter, 27 (i.e., $3 \times 3 \times 3$ ) different scenarios could be defined for each dozing distance of each model. For example, a scenario for a machine with dozing distance of $15 \mathrm{~m}$ can be: to work with blaster rocks in a good working condition on a grade of $+15 \%$. Accordingly, 26 other scenarios could be considered for this machine. In order to construct and study all scenarios for all dozers, during the 8-month period of time, all models were separately tracked to collect all required data. 


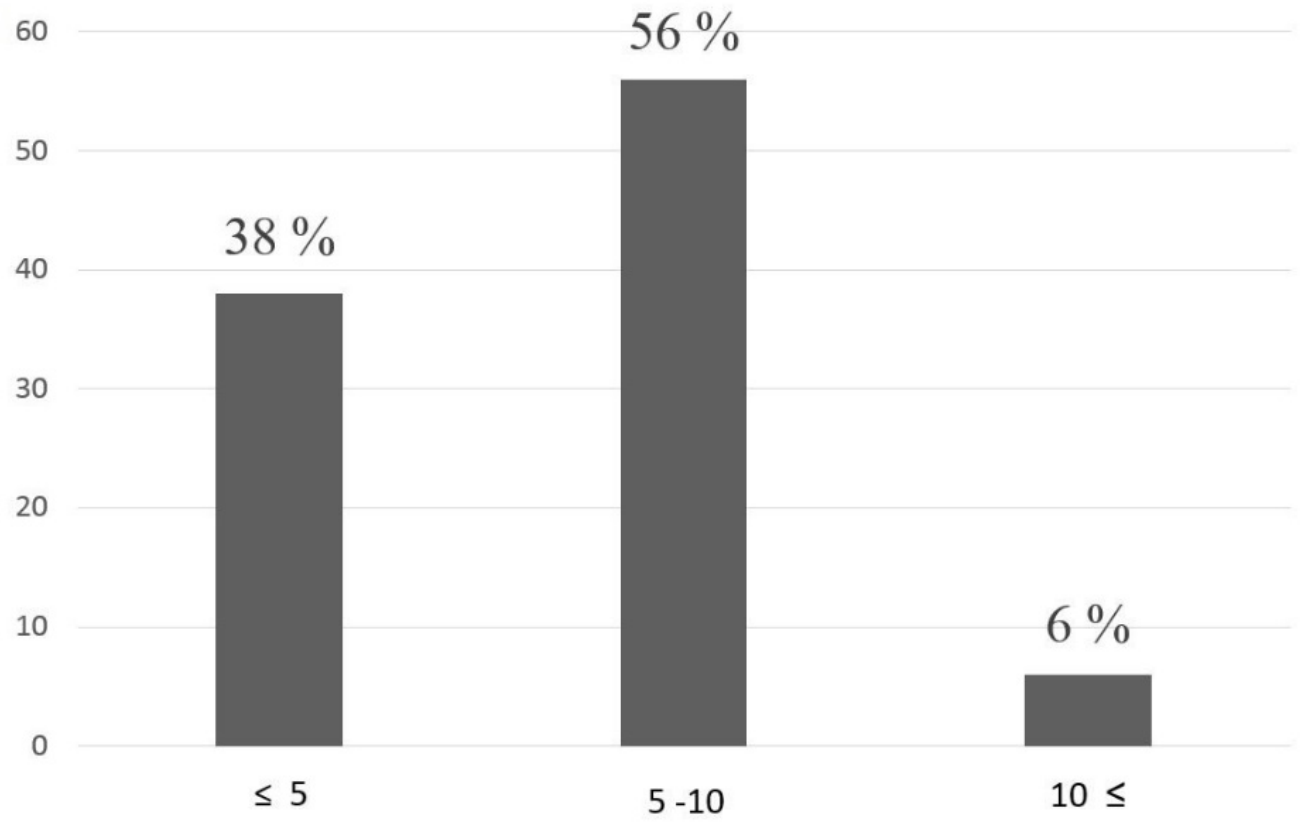

Figure 2. Working experience (years) of machine operators

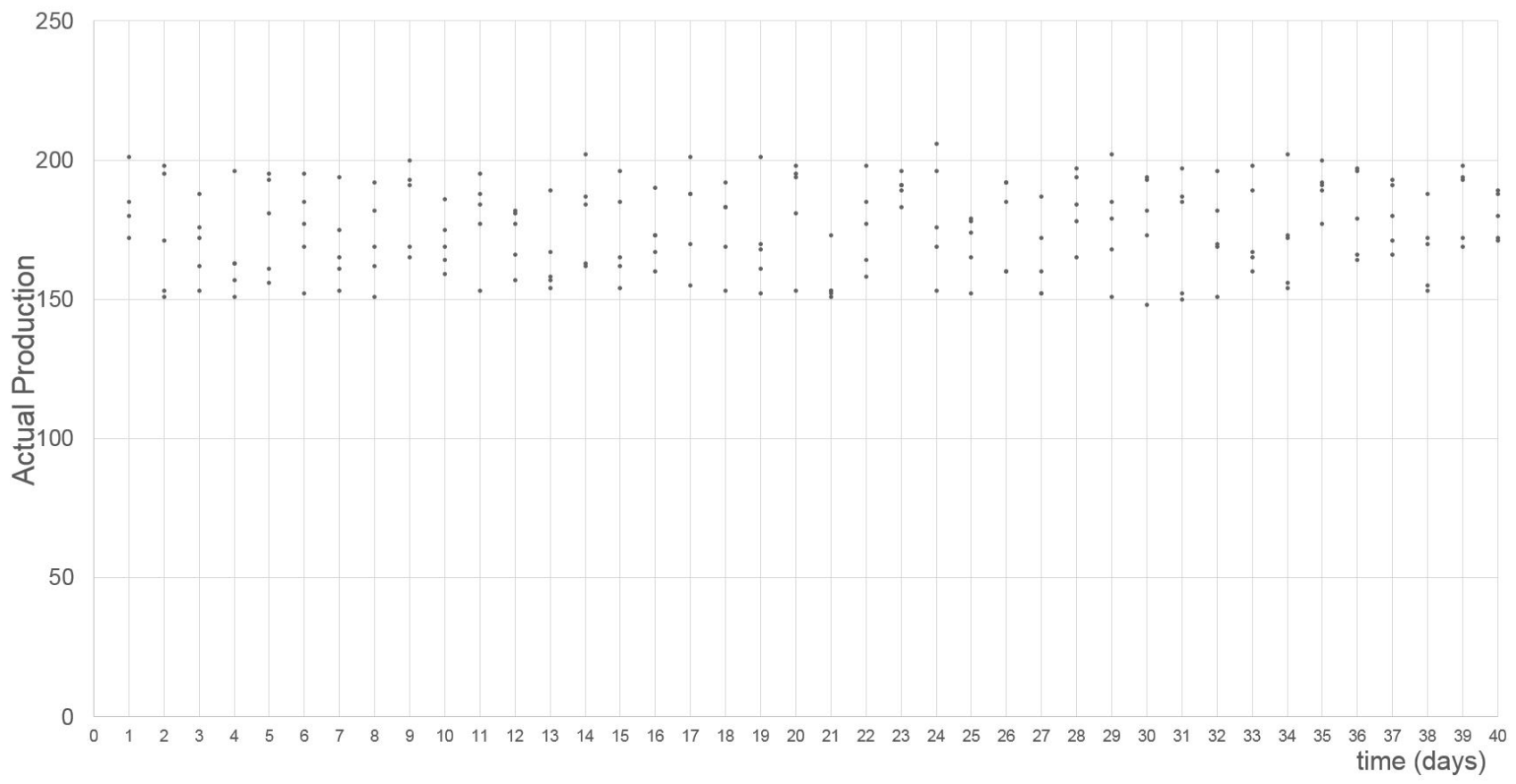

Figure 3. A sample set of real data

\subsection{Data Analysis}

After collecting the required actual data, appropriate statistical analyses were employed to find best representative for each scenario of each model. In this context, for each individual scenario a sample of 40 data was collected for each individual project. These samples were not collected in consecutive days; collecting data in consecutive days might provide opportunity for happening the bias in data. In addition, the minimum number of data more than 30 for each sample could help that the sample set would be approximately normal [37-41]. Such data sets would be suitable to conduct statistical analysis with reliable results [42-47]. Since there were 5 borrow pits in the case of this research, 200 (i.e., $5 \times 40$ ) samples for each scenario of each model were finally collected. Figure 3 presents a sample set of the data collected for model D7R of Caterpillar dozer with dozing distance of $30 \mathrm{~m}$. This samples shows that actual hourly production data ranges approximately from 150 to $200 \mathrm{~m}^{3}$ that shows a $25 \%$ difference in the range of data.

According to the classification of the collected data, One-Way ANOVA was selected and employed to analyze the data and find a representative for each scenario. OneWay ANOVA is a numerical statistical method for comparing three or more independent sample groups in order to find whether the differences between means of the groups are statistically significant or not. In other words, this method is typically used to statistically understand the differences among independent groups of 
samples. In this research, the One-Way ANOVA tested a hypothesis on sample means for each scenario of individual dozers. In this context, the null and alternative hypotheses are as follow:

- Null hypothesis: There are no statistically significant differences among means of 5 sample sets for each model of dozer.

- Alternative hypothesis: There are statistically significant differences among means of 5 sample sets for each model of dozer.

In the context of One-Way ANOVA, when the null hypothesis is not rejected, the grand mean (i.e. the mean for all samples) is selected as representative for all the data. In this research, the null hypothesis was tested through five independent groups of 40 samples for each machine. Table 2, as an example, shows the results for One-Way ANOVA analysis employed on the data collected for Caterpillar model D7R with dozing distance of $30 \mathrm{~m}$ (see Figure 3). In this example, F-value is less than the critical value, and therefore the null hypothesis was failed to be rejected. In other words, the result indicates that the grand mean is the representative for the data of this scenario. Accordingly, all data sets for all models were tested, and for all them the null hypothesis was failed to be rejected; for few machines the null hypothesis was marginally failed to be rejected. It is worth mentioning that all estimations and tests were performed in the opensource statistical language $\mathrm{R}$.

Table 2. Sample of One-Way ANOVA Analysis

\begin{tabular}{cccccc}
\hline Source & df & SS & MS & F & Critical Value \\
\hline Model & 4 & 2401.93 & 600.48 & 2.54 & 3.42 \\
Error & 195 & 46086.15 & 236.33 & & \\
Total & 199 & 48488.08 & & & \\
\hline
\end{tabular}

\section{Results}

Based the One-Way ANOVA analysis, the best representative for each scenario of the three assigned dozing distances for each model was finally found.

In this research, it was decided to present the results in practical implications (e.g., see Figure 1). In this context, therefore, the author first chose one of the scenarios as the base scenario. Then, by finding the correlations among the base and other scenarios, the correction factors which adjust the results of the base scenario to other scenarios were estimated. It is worth mentioning that manufacturers generally use this method to present production of their machinery and equipment. In particular, this method for presenting the results mainly helps better understanding the discrepancies between nominal and actual hourly production.

In this context, the scenario in which machines work with loose soil in good condition on ground with zero slope was chosen as the base scenario for all models.
Table 3 and Table 4 provide the results of One-Way ANOVA for this scenario. Figure 4 and Figure 5 also show the production charts for the data provided in Table 3 and Table 4, respectively. Based on the found three points for each model, the best curve was fitted to the points. Compared to Figure 1, Figure 4 and Figure 5 present the actual hourly production for Caterpillar and Komatsu dozers, respectively. For models of Caterpillar dozer presented in Figure 4, by increasing the dozing distance, the rate of production for model of $\mathrm{B}$ and $\mathrm{C}$ decreases by an amount significantly higher than that of for $\mathrm{F}, \mathrm{G}$, and $\mathrm{H}$. However, this rate remains approximately the same, specifically for dozing distance more than $30 \mathrm{~m}$, for all models of Komatsu (see Figure 5).

Table 3. Actual Hourly Production for Models of Caterpillar

\begin{tabular}{ccc}
\hline Model & $\begin{array}{c}\text { Dozing } \\
\text { Distance(m) }\end{array}$ & Actual Hourly Production $\left(\mathbf{L m}^{3} \mathbf{h} \mathbf{h r}\right)$ \\
\hline \multirow{2}{*}{ D6N (H) } & 15 & 149 \\
& 30 & 101 \\
& 75 & 43 \\
\hline D6T (G) & 15 & 281 \\
& 30 & 144 \\
& 75 & 62 \\
\hline \multirow{2}{*}{ D7R (F) } & 15 & 305 \\
& 30 & 174 \\
& 75 & 86 \\
\hline \multirow{2}{*}{ D9T (C) } & 15 & 912 \\
& 30 & 536 \\
& 75 & 289 \\
\hline D10T (B) & 15 & 1353 \\
& 30 & 738 \\
& 75 & 304 \\
\hline
\end{tabular}

Table 4. Actual Hourly Production for Models of Komatsu

\begin{tabular}{ccc}
\hline Model & $\begin{array}{c}\text { Dozing } \\
\text { Distance(m) }\end{array}$ & Actual Hourly Production $\left(\mathbf{L m}^{3} / \mathbf{h r}\right)$ \\
\hline 15 & 527 \\
\hline & 30 & 290 \\
& 75 & 95 \\
\hline D155A-6 & 15 & 746 \\
& 30 & 351 \\
& 75 & 140 \\
\hline D275A & 15 & 1041 \\
& 30 & 571 \\
& 75 & 270 \\
\hline D375A & 15 & 1759 \\
& 30 & 915 \\
& 75 & 450 \\
\hline
\end{tabular}




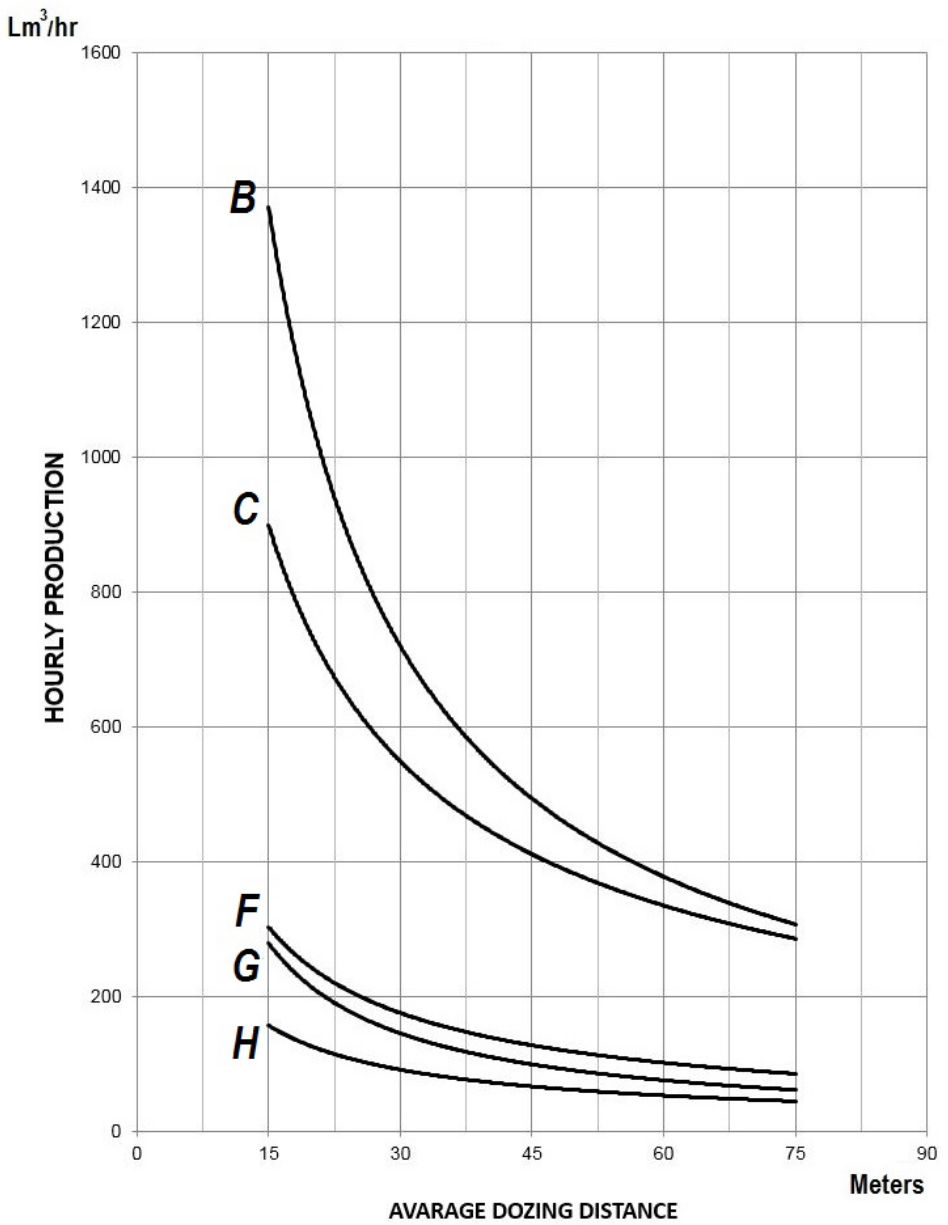

Figure 4. Chart for actual hourly production for models of Caterpillar

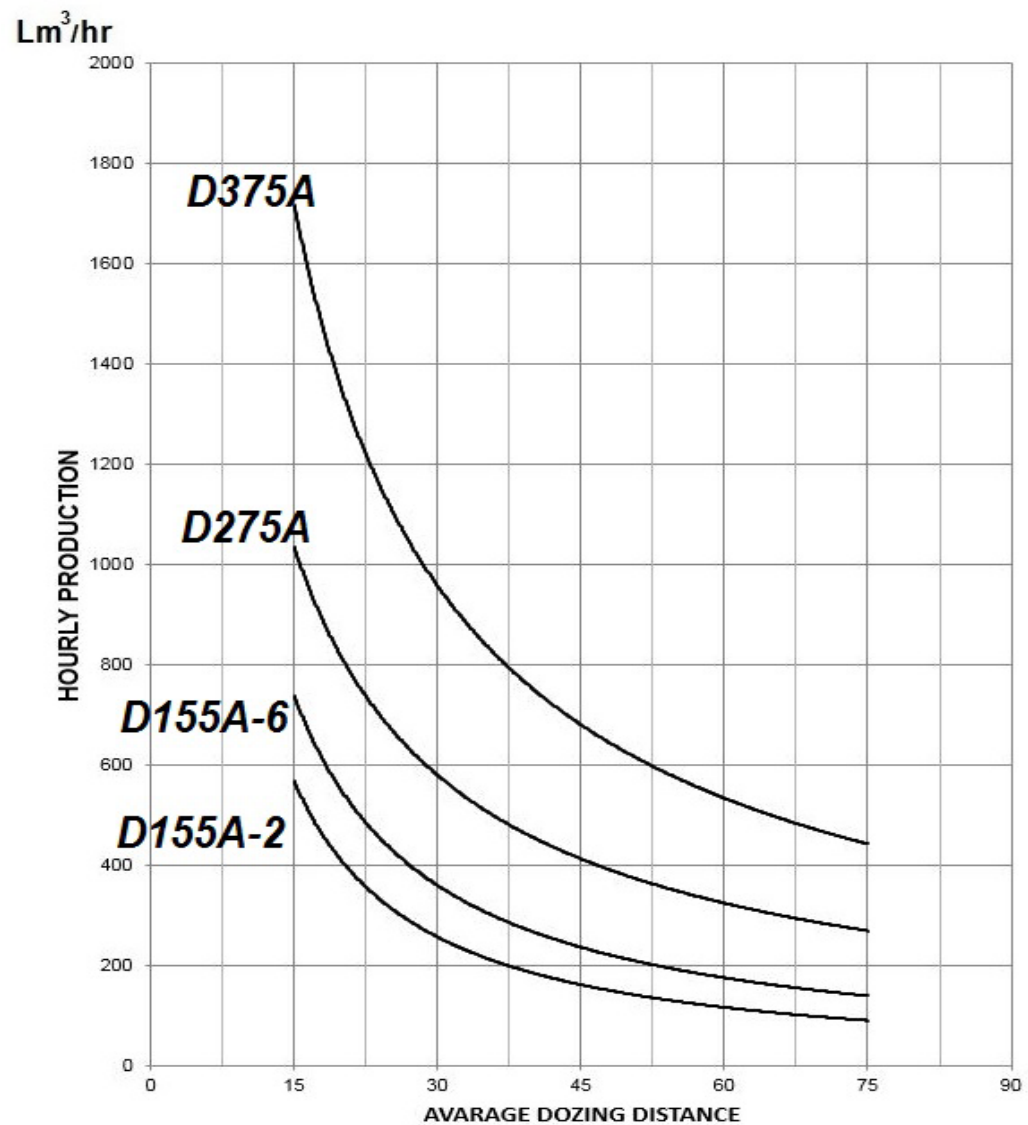

Figure 5. Chart for actual hourly production for models of Komatsu 
Furthermore, Table 5, Table 6, and Figure 6 present the correction factors which should be employed to achieve the results for the other scenarios. These correction factors were also resulted from One-Way ANOVA analysis. Depending on the project site operating conditions (i.e., working condition, material condition, and ground slope), the appropriate correction factors might be applied to the charts of actual hourly production data (see Figure 4 and Figure 5) to estimate the production of interest. In particular, the formula for production calculation is:

\section{Estimated Actual Hourly Production$$
\text { = Actual Hourly Production from Charts }
$$$$
\times(\Pi \text { Correction Factors })
$$

For example, a Caterpillar model D7R (i.e., F) assumed works in a project site with the following conditions:

(1) Dozing distance: $30 \mathrm{~m}$.

(2) Working condition: Medium

(3) Materials: Blaster rocks

(4) Ground slope: +10

The data and correction factors for aforementioned conditions are as follow, respectively:
(1) $174\left(\mathrm{~m}^{3} / \mathrm{hr}\right)$ (see Table 3)
(2) 0.60
(3) 0.52
(4) 0.70
(see Table 5)
(see Table 6)
(see Figure 6)

Therefore, the machine production is: $174\left(\mathrm{~m}^{3} / \mathrm{hr}\right) \times(0.60$ $\times 0.52 \times 0.70)=38\left(\mathrm{~m}^{3} / \mathrm{hr}\right)$

This example shows a big discrepancy between nominal and actual hourly production which can be happen in a real construction project due to different project site conditions. It is worth mentioning that Table 5 presents a range for correction factors related to the working conditions. In this context, choosing a number form this range is mainly depends on the machinery manager's decisions in a project site. For this example, 0.60 was chosen from the range from 0.52 to 0.67 provided for medium working conditions of the dozer.

In addition, for most of the correction factors provided through Table 5, Table 6, and Figure 6, the amount of Komatsu factors are higher than that of Caterpillar factors which leads to higher production in a same project site condition. Compared to the Komatsu dozers, the Caterpillar models worked 6 years on average more; it could be considered as the main reasons for lower production. However, Table 6 shows that for blaster rocks, a Caterpillar dozer can work better than a Komatsu dozer. This might lead to concluding that Caterpillar dozers typically work with blaster rocks better than Komatsu Dozers. In addition, Figure 6 shows for downhills, a model of Caterpillar could work better than a Komatsu model. The effect of gravitational force helps movement of a machine in on downward slopes [3]. This effect named grade assistance is resulted from machine weight, and it is consistently independent from machine age.

Table 5. Correction Factors for Working Conditions

\begin{tabular}{ccc}
\hline Working Condition & Caterpillar & Komatsu \\
\hline Good & $0.83 \sim 0.61$ & $0.83 \sim 0.77$ \\
Medium & $0.67 \sim 0.52$ & $0.67 \sim 0.59$ \\
Weak & $0.50 \sim 0.36$ & $0.50 \sim 0.44$ \\
\hline
\end{tabular}

Table 6. Correction Factors for Materials

\begin{tabular}{ccc}
\hline Type of Materials & Caterpillar & Komatsu \\
\hline Loose soil & 1.00 & 1.00 \\
Soil + Rubble stones & 0.62 & 0.69 \\
Blaster rocks & 0.52 & 0.46 \\
\hline
\end{tabular}

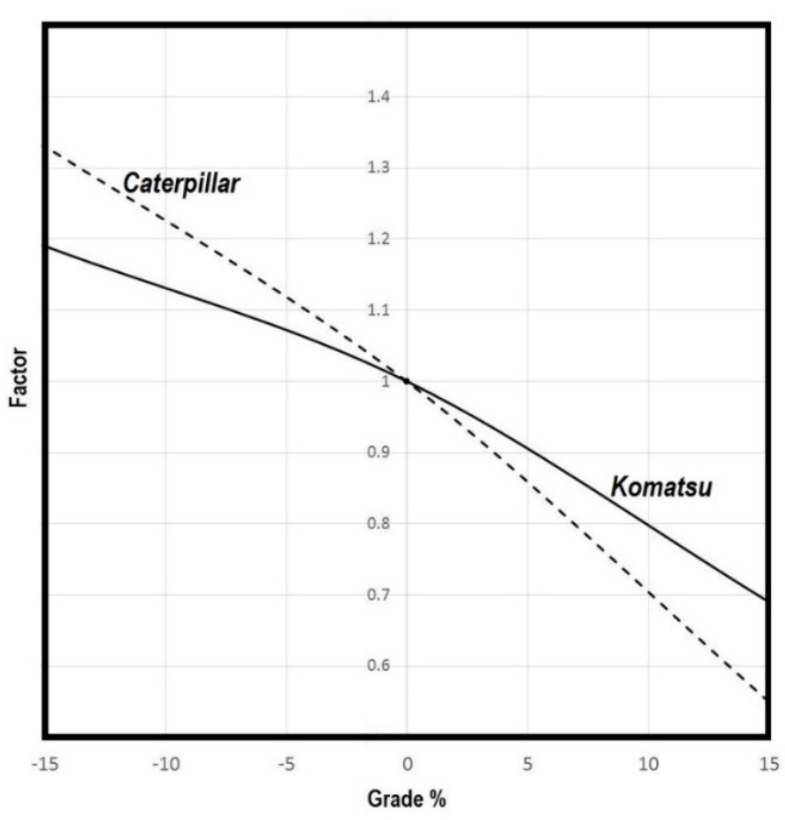

Figure 6. Correction factors for ground slope

\section{Discussion}

Caterpillar and Komatsu manufacturers regularly provide a new version of their own catalogues for their customers. The catalogues includes different charts, tables, and figures to show how their machines and equipment works in ideal conditions; such assumed ideal conditions could be hardly achieved on a real project.

This paper investigated the actual production of crawler-type dozers that work in borrow pits. The main parameters which affect a machine production were also investigated. A comparison between estimated results from this study and manufacturers' results shows (see Figure 7 and Figure 8) a critical discrepancy between the nominal and actual production in the ideal project site conditions for all models of dozers; an average drop of $500 \mathrm{Lm}^{3} / \mathrm{hr}$ exist for Caterpillar models at dozing distance of $15 \mathrm{~m}$. In addition, Figure 7 shows that by increasing the dozing distance, the rate of change for both nominal and actual productions remains approximately the same for model B of Caterpillar, while this rate for actual production of models $\mathrm{C}, \mathrm{F}, \mathrm{G}$, and $\mathrm{H}$ is lower than that of nominal production. In other words, compared to the dozing distance of $15 \mathrm{~m}$, there is not such a big discrepancy in production for models of $\mathrm{C}, \mathrm{F}, \mathrm{G}$, and $\mathrm{H}$ at dozing distance of $75 \mathrm{~m}$. Furthermore, Figure 8 shows that for all models of Komatsu studied in this research, by increasing the dozing distance, the rate of change in production is lower for actual production than nominal production. 


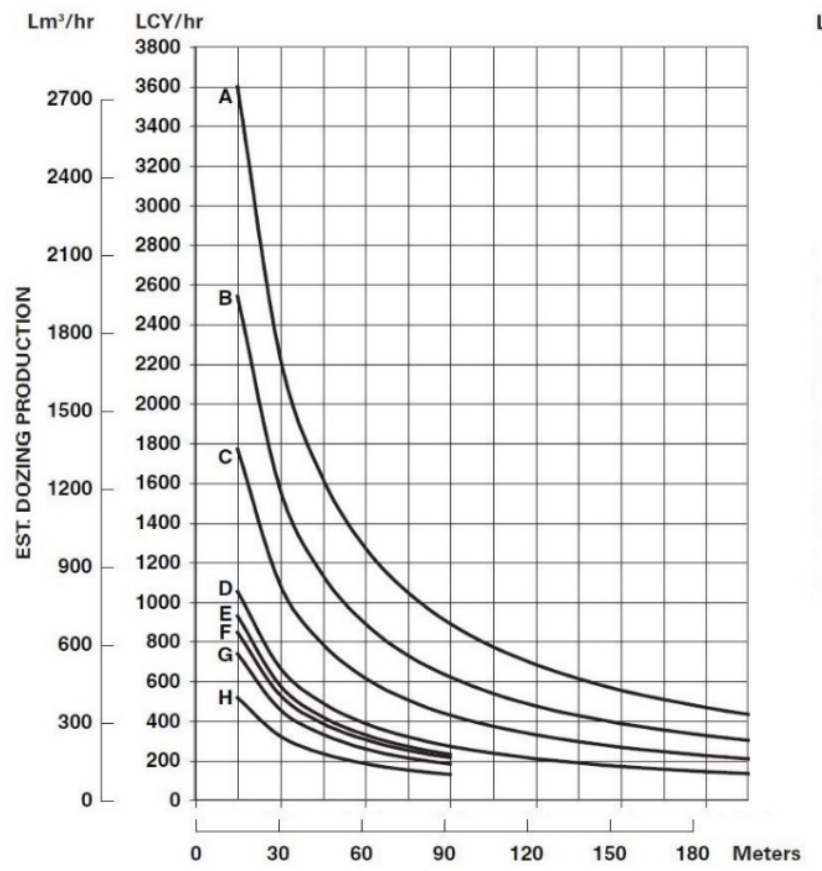

AVERAGE DOZING DISTANCE

Manufacturer Chart

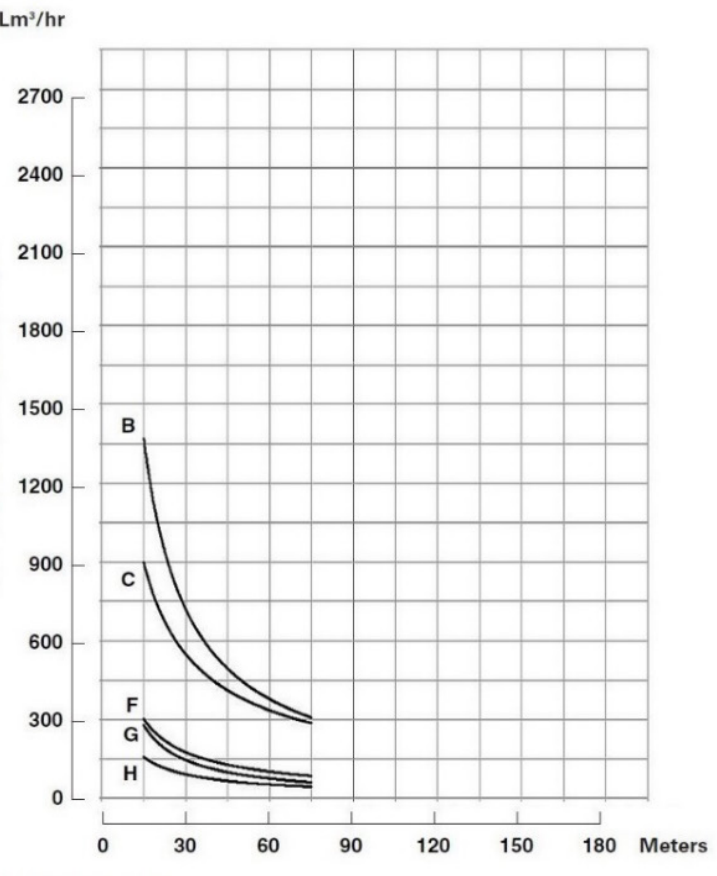

Figure 7. Charts for nominal and actual data for models of Caterpillar
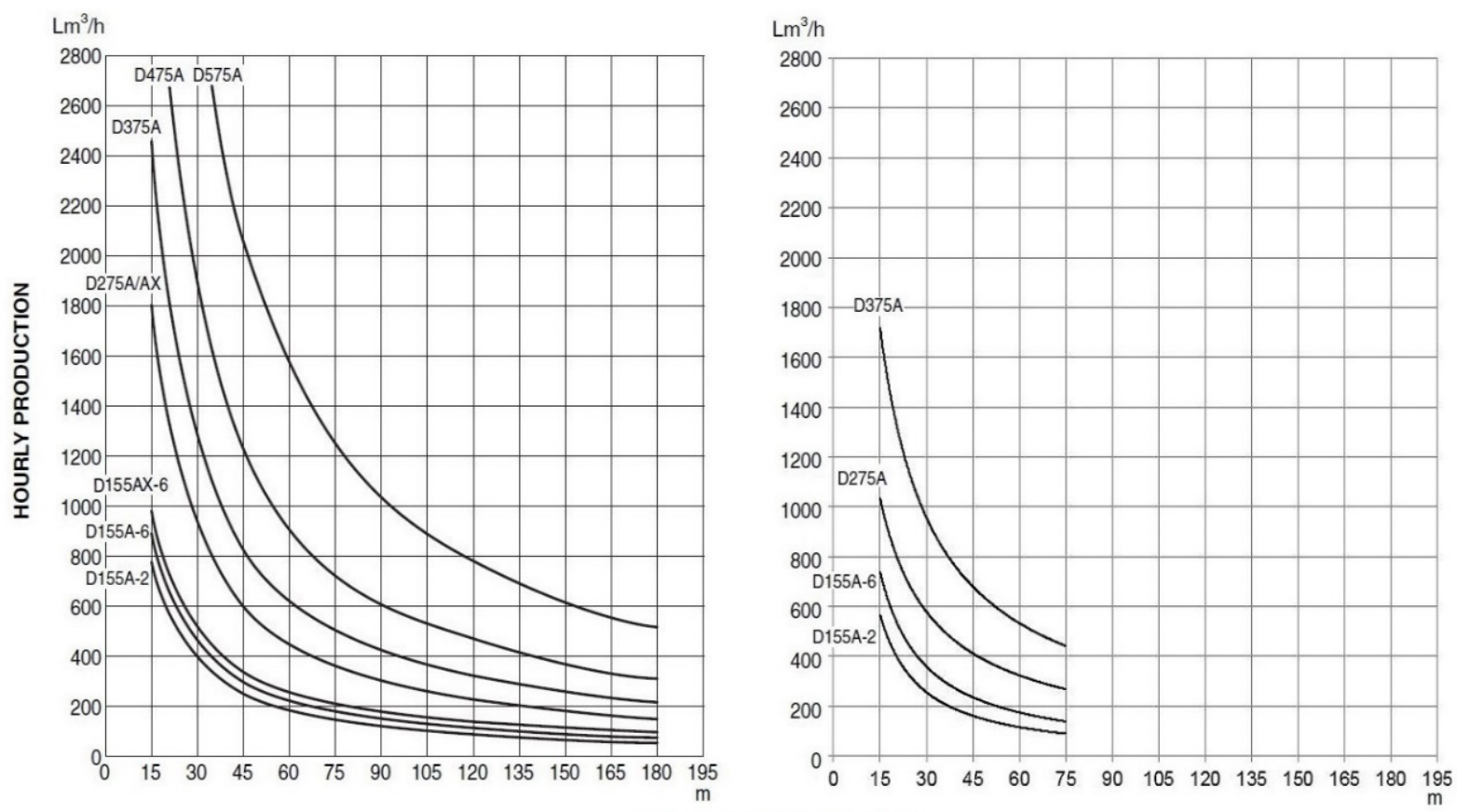

Manufacturer Chart

AVERAGE DOZING DISTANCE

Figure 8. Charts for nominal and actual data for models of Komatsu

Table 7 and Table 8, and Figure 9 present the differences between the estimated correction factors and the factors provided by two manufacturers. Table 7 shows that this study results present a range for correction factors for models of Komatsu, while the manufacturer presents a point estimation for factors; considering a range for correction factors could be more logical. In addition, for all working conditions of Caterpillar dozers, the range for estimated correction factors is within the limits of ranges provided by the manufacturer (see Table 7). Table 8 presents the correction factors for different types of materials. Since the estimated limits for range was too short, a point estimation is provided. In addition, Table 8 shows that for the four ranges provided by manufacturers' data, the estimated correction factors are within the limitation of ranges. Figure 9 presents that for all models of Caterpillar and Komatsu dozers work on a downhill slope, the estimated correction factors for ground slope is approximately as same as the factors provided by the manufacturers. As discussed, since the gravitational force helps movement of a machine in on downward slopes, the correction factors remains the same. On the contrary, the 
engine power of a machine plays the critical role when a machine works on upward slopes. Whereas the dozers studied in this research were not new models, there is a significant discrepancy between correction factors provided by manufacturers with those provided by this study. In particular, since the average working age of Caterpillar models is more than that of Komatsu models, this discrepancy is higher for Caterpillar models (see Figure 9).

Table 7. Correction Factors for Working Conditions

\begin{tabular}{ccccc}
\hline Working & \multicolumn{2}{c}{ Obtained Results } & \multicolumn{2}{c}{ Manufacturers Data } \\
\cline { 2 - 5 } Condition* & Caterpillar & Komatsu & Caterpillar & Komatsu \\
\hline Good & $0.83 \sim 0.61$ & $0.83 \sim 0.77$ & $0.83 \sim 0.49$ & 0.83 \\
Medium & $0.67 \sim 0.52$ & $0.67 \sim 0.59$ & $0.67 \sim 0.40$ & 0.67 \\
Weak & $0.50 \sim 0.36$ & $0.50 \sim 0.44$ & $0.50 \sim 0.30$ & 0.50 \\
\hline
\end{tabular}

*The definition of working condition has some changes with the manufacturer definition.

Table 8. Correction Factors for Materials

\begin{tabular}{ccccc}
\hline Type of & \multicolumn{2}{c}{ Obtained Results } & \multicolumn{2}{c}{ Manufacturers Data } \\
\cline { 2 - 5 } Materials* & Caterpillar & Komatsu & Caterpillar & Komatsu \\
\hline Loose soil & 1.00 & 1.00 & 1.00 & $1.00 \sim 0.81$ \\
Soil + Rubble & 0.62 & 0.69 & 0.67 & $0.81 \sim 0.67$ \\
stones & 0.52 & 0.46 & $0.67 \sim 0.50$ & $0.50 \sim 0.36$ \\
Blaster rocks & & & & \\
\hline
\end{tabular}
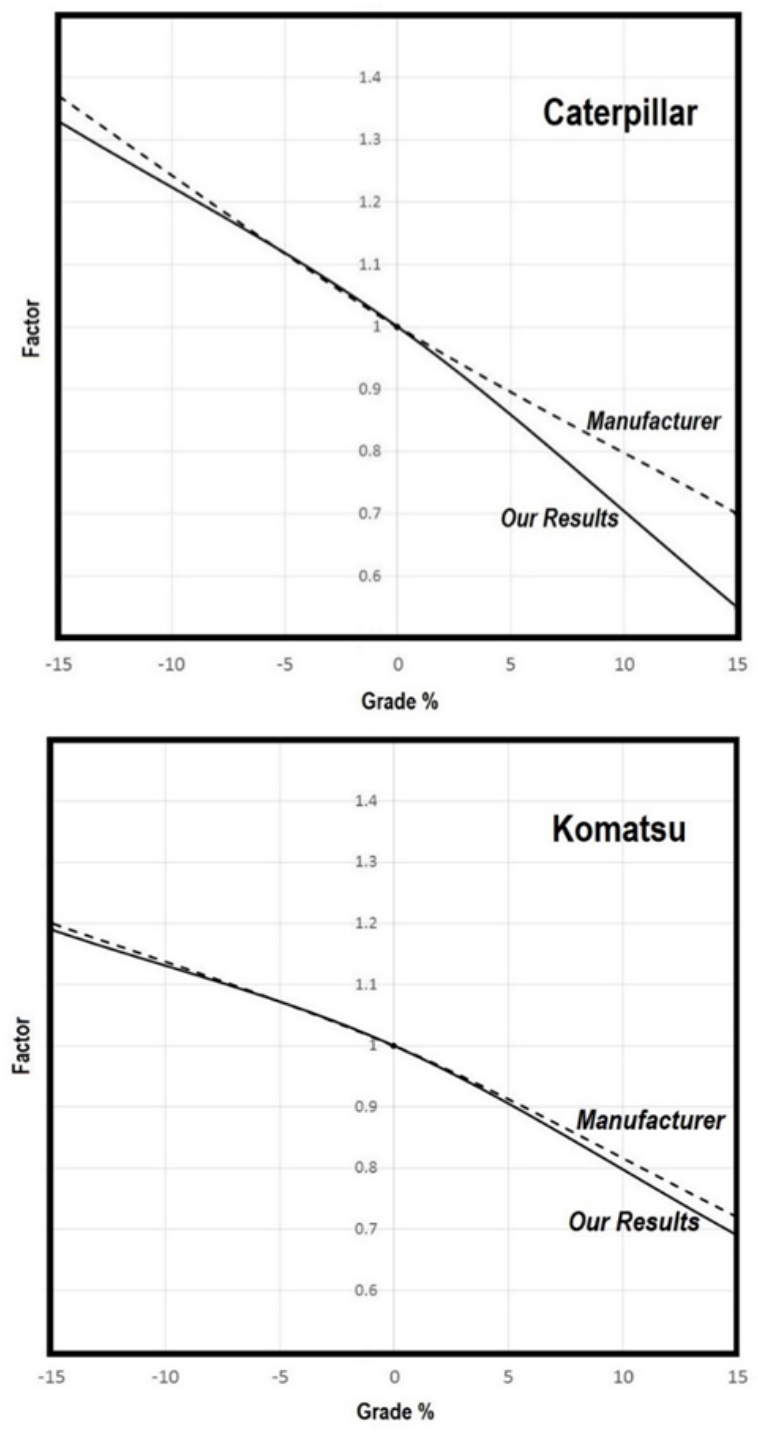

Figure 9. Correction factors for ground slope
In summary, these results shows that for different project sites conditions, there is a significant discrepancy between actual and nominal hourly production (see Figure 7 and Figure 8), while the correction factors mostly remain unchanged (see Table 7 and Table 8, and Figure 9).

The working condition considered in this study as a parameter which includes the effect of machinery age, operators' skills, and weather condition. The manufactures' charts are on the basis of ideal condition that for such conditions, a machine is typically considered as a new machine, the operator is considered as a fully-skilled one, and the weather condition is ideal. However, in real project sites, achieving to these ideal conditions rarely occurs. In the presented case study for this research, there were no new machines (see Table 1), and the machine operators always were not the best fully skilled operators (see Figure 2). These two sub parameters of working condition, therefore, could lead to significant discrepancies between nominal and actual hourly production (see Figure 7 and Figure 8).

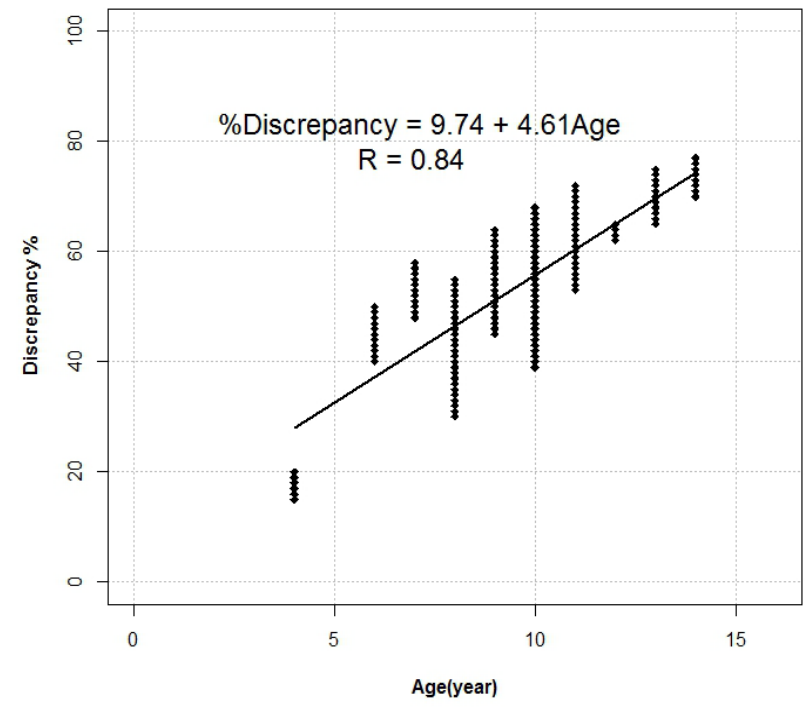

Figure 10. Linear regression model for models of Caterpillar

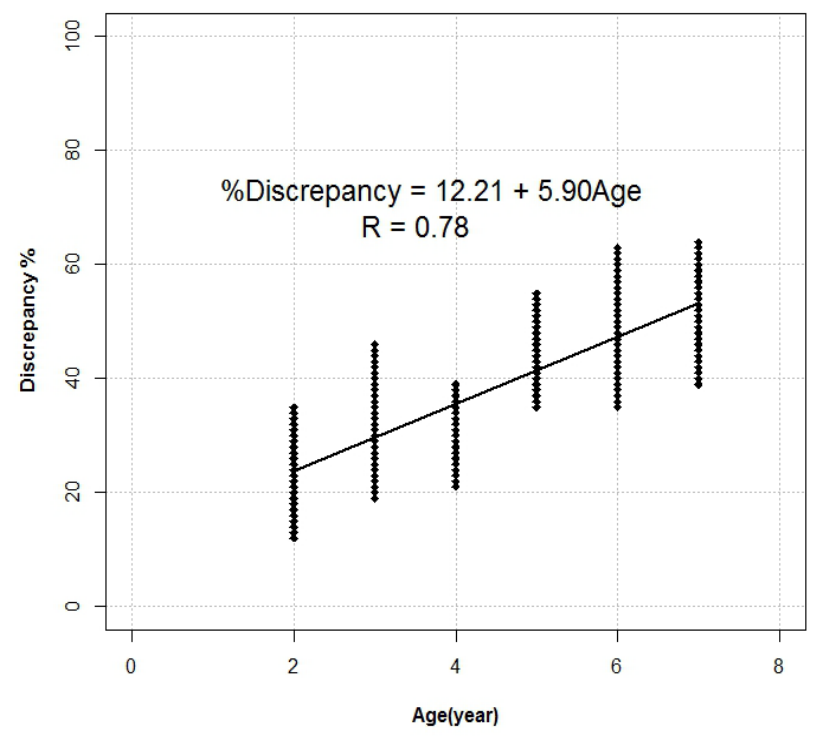

Figure 11. Linear regression model for models of Komatsu 
Table 9. Results of Linear Regression Models

\begin{tabular}{|c|c|c|c|c|c|c|}
\hline & Coefficient & Estimated Coefficient & Standard Error & T-Value & P-Value & $\mathbf{R}$ \\
\hline \multirow{2}{*}{ Caterpillar } & (Intercept) & 9.74345 & 0.41005 & 22.8 & $<2 \times 10^{-16}$ & \multirow{2}{*}{0.8413} \\
\hline & Age & 4.61067 & 0.03772 & 123.0 & $<2 \times 10^{-16}$ & \\
\hline \multirow{2}{*}{ Komatsu } & (Intercept) & 12.21153 & 0.34291 & 37.05 & $<2 \times 10^{-16}$ & \multirow{2}{*}{0.7791} \\
\hline & Age & 5.90495 & 0.07833 & 73.37 & $<2 \times 10^{-16}$ & \\
\hline
\end{tabular}

In addition, a statistical comparison between the machinery age and discrepancies between nominal and actual production can illustrate how the age of a machine might affect its production. This comparison could address the third sub-objective of this research study. In particular, therefore, simple linear regression models are developed to estimate a mathematical relationship between machinery age and discrepancy for different models of Caterpillar and Komatsu. In fact, these regression models help understand how a machine age affect is production discrepancy. In this context, for the regression models, the machine age and percentage of discrepancy are considered as explanatory and response variables, respectively. Figure 10 and Figure 11 presents the developed regression models for dozers of Caterpillar and Komatsu, respectively. It is worth mentioning that all data for dozers points out on Figure 10 and Figure 11 to show discrepancy range found for all model with the same working years. For example, Figure 10 shows that this range is approximately between $38 \%$ and $67 \%$ for models of Caterpillar with 10 working years.

Table 9 shows the results of linear regression models. The results show that two variables are positively associated for each regression model, and the amount of p-values and Pearson correlation coefficients (i.e., R) indicates the strength of this associated relationship, specifically for Caterpillar dozers. Therefore, it can be concluded that the age of a machine could help predict its production's discrepancy and therefore, to predict its actual hourly production.

In addition to the aforementioned discussion, compared to the Rashidi et al. [23] and Ok and Sinha [26] research which studied actual productions of dozer, this research provides real charts for productions and indicates how the correction factors are affected in various project sites. In addition, to best knowledge of the author, it is the first research work that presents all the results in the format of manufacturers' publication. Such results provide a critical unique opportunity to visually understand the differences between nominal and actual hourly production when a machine works in a project site.

\section{Limitations and Recommendation for Future Research}

The results and discussion on the results could address the objectives and sub-objectives of this study. However, this research is also subjects to some limitations, as follow:

1. This study's findings and results are based on a case study for dozers working at five borrow pits. Such results might not be extended to other types of projects in where crawler-type dozer are utilized for a different job; the machine also can be used for land clearing in a project site. It could be considered as the main limitation for this work.

Future research is recommended to study crawler type dozer in different working sites besides dozing in order to understand how the machine acts for such working conditions.

2. There are uncertainties in collected data. Such uncertainties specifically can happen during data collection steps and therefore could affect the results.

It is recommended that future research employs various machine learning methods such as neural network, and statistical methods such as multivariate analysis in order to find and void such uncertainties.

3. Operators were assigned randomly to different models of dozer.

In such work, future research is recommended to consider a plan in order to assign an operator to a machine. Such plans could provide an opportunity to understand how operators' skills might affect machine production.

4. Climate conditions and annual rainfall were assumed to be equal for the five borrow pits. Therefore, the effects of these parameters were neglected for this study.

Future research might study the effects of climates conditions and annual rainfall as part of the working condition in order to achieve more realistic results and conclusions.

It is worth mentioning that in such research work, the data collection step is the main step which leads to achieving reliable results and conclusions. This step significantly depends on the aim of a study. In fact, the aim of study should to be clearly defined before starting to collect required data. In particular, the aim determines not only the required time for data collection step but also the methodologies needed to be employed to collect required data. In most cases, the data collection step is extremely time consuming. Future work is therefore recommended to employ remote sensing data methodologies to collect required data. These methodologies are expected to save required time and cost needed for the data collection step.

\section{Conclusion}

This paper employed the One-Way ANOVA analysis and linear regression model to understand and estimate the discrepancies between actual and nominal production for crawler-type dozer. In order to collect the required data for this research, 21 different models of Caterpillar with average working year of 10.1 and 18 different models of 
Komatsu with average working year of 4.1 were individually considered in five different borrow pits for an eight-month period of time. For each machine, three different dozing distances were considered, and actual hourly production was estimated based on the ANOVA analysis at each dozing distance. The results show significant reductions (i.e., discrepancies) in machine hourly production rates; these discrepancies should to be considered in planning of machinery and equipment. In particular, the results from linear regression models indicate that a linear relationship could exist between working age of a machine and its production discrepancies. Such relationship could help estimate the actual production of a machine based on its working age. In addition, this paper also studied three parameters that affect a machine production in a project site. The correction factors for each individual parameter were found. These correction factors approximately remained in the range provided by manufacturers.

The author believe that the results of this study could provide appropriate prospect for machinery managers in construction sites; the results present promising options for future research into machinery and equipment production.

\section{Disclaimer}

The opinions, findings, and conclusions expressed in this paper are those of the authors and do not necessarily prove or disprove the view of Caterpillar and Komatsu.

\section{List of Abbreviations and Symbols}

$\begin{array}{ll}\mathrm{Lm}^{3} & \text { Loose cubic meters } \\ \mathrm{Lm}^{3} / \mathrm{h} & \text { Loose cubic meters per hour } \\ \mathrm{Lm}^{3} / \mathrm{hr} & \text { Loose cubic meters per hour } \\ \mathrm{Lyd}^{3} / \mathrm{h} & \text { Loose cubic yards per hour } \\ \mathrm{LCY} / \mathrm{hr} & \text { Loose cubic yards per hour } \\ \mathrm{ft} & \text { Feet } \\ \mathrm{m} & \text { Meter } \\ \mathrm{hp} & \text { Engine horse power }\end{array}$

\section{References}

[1] D. Gransberg, C. Popescu, R. Ryan, Construction Equipment Management for Engineers, Estimators, and Owners, Taylor \& Francis, 2006.

[2] K. Samee, J. Pongpeng, Structural equation model for construction equipment management affecting project and corporate performance, KSCE J. Civ. Eng. (2015) 1-15.

[3] R. Peurifoy, C. Schexnayder, A. Shapira, R. Schmitt, Construction Planning, Equipment, and Methods, 8th ed., McGraw-Hill, 2010.

[4] Building Intelligent Applications for Construction Equipment Management, in: Comput. Civ. Eng. 2007, American Society of Civil Engineers, n.d.: pp. 192-199.

[5] O. Tatari, M. Skibniewski, Integrated agent - based construction equipment management: Conceptual design, J. Civ. Eng. Manag. 12 (2006) 231-236.

[6] G. Heravi, H.N. Rafsanjani, Critical Safety Factors in Construction Projects, Adv. Mater. Res. 255-260 (2011) 3921-3927.

[7] H.N. Rafsanjani, A Computer Model for Selecting Equipment in Earth-Fill Dam Projects, J. Civ. Eng. Archit. 5 (2011) 465-470.
[8] H.N. Rafsanjani, An assessment of nominal and actual hourly production of crawler-type front shovel in construction project, JCE-IEB. 40 (2012) 115-124.

[9] H.N. Rafsanjani, Comparison between Nominal and Actual Hourly Production of Crawler-Type Dozer: A Case Study, in: Comput. Civ. Eng. 2015, American Society of Civil Engineers, n.d.: pp. 17-24.

[10] H.N. Rafsanjani, Y. Gholipour, H.H. Ranjbar, An Assessment of Nominal and Actual Hourly Production of the Construction Equipment Based on Several Earth-Fill Dam Projects in Iran, Open Civ. Eng. J. 3 (2009) 74-82.

[11] H.N. Rafsanjani, S. Shahrokhabadi, A. Hadjahmadi, The Use of Linear Regression to Estimate the Actual Hourly Production of a Wheel-Type Loader in Construction Projects, in: ICSDEC 2012, American Society of Civil Engineers, n.d.: pp. 727-731.

[12] S.W. Nunnally, Managing construction equipment, 2nd ed., Prentice-Hal, 2000.

[13] Modeling the Dynamics of Heavy Equipment Management Practices and Downtime in Large Highway Contractors, J. Constr. Eng. Manag. 135 (2009) 939-947.

[14] G. Lucko, A Statistical Analysis and Model of the Residual Value of Different Types of Heavy Construction Equipment, 2003.

[15] A. Tsimberdonis, E. Murphree, Equipment Management through Operational Failure Costs, J. Constr. Eng. Manag. 120 (1994) 522-535.

[16] C. Schexnayder, HEAVY CONSTRUCTION EQUIPMENT REPLACEMENT ECONOMICS, 1980. http://docs.lib.purdue.edu/dissertations/AAI8102704/.

[17] A. Tavakoli, J. Masehi, C. Collyard, FLEET: Equipment Management System, J. Manag. Eng. 6 (1990) 211-220.

[18] H. Tajeen, Z. Zhu, Image dataset development for measuring construction equipment recognition performance, Autom. Constr. 48 (2014) 1-10.

[19] N. Pradhananga, J. Teizer, Automatic spatio-temporal analysis of construction site equipment operations using GPS data, Autom. Constr. 29 (2013) 107-122.

[20] H. Yip, H. Fan, Y. Chiang, Predicting the maintenance cost of construction equipment: Comparison between general regression neural network and Box-Jenkins time series models, Autom. Constr. 38 (2014) 30-38.

[21] A. Hajji, The use of construction equipment productivity rate model for estimating fuel use and carbon dioxide (CO2) emissionsCase study: bulldozer, excavator and dump truck, Int. J. Sustain. Eng. 8 (2015) 111-121.

[22] Comparison of Two Models for Estimating Equipment Productivity for a Sustainability Quantification Tool, in: ICSDEC 2012, American Society of Civil Engineers, n.d.: pp. 626-633.

[23] A. Rashidi, H.R. Nejad, M. Maghiar, Productivity estimation of bulldozers using generalized linear mixed models, KSCE J. Civ. Eng. 18 (2014) 1580-1589.

[24] S. Han, T. Hong, S. Lee, Production prediction of conventional and global positioning system-based earthmoving systems using simulation and multiple regression analysis, Can. J. Civ. Eng. 35 (2008) 574-587.

[25] K. Schabowicz, B. Hola, Mathematical-neural model for assessing productivity of earthmoving machiner, J. Civ. Eng. Manag. 13 (n.d.) 47-54.

[26] S.C. Ok, S.K. Sinha, Construction equipment productivity estimation using artificial neural network model, Constr. Manag. Econ. 24 (2006) 1029-1044.

[27] C.D. Edmonds, B. Tsay, W. Lin, Analyzing machine efficiency, Natl. Public Account. 39 (1994) 28-44.

[28] J. Zou, HSV Color-space Digital Image Processing for the Analysis of Construction Equipment Utilization and for the Maintenance of Digital Cities Image Inventory, University of Alberta (Canada), 2006.

[29] G.P. Hammer, J.-B. du Prel, M. Blettner, Avoiding Bias in Observational Studies, Dtsch. Ärztebl. Int. 106 (2009) 664-668.

[30] C.J. Pannucci, E.G. Wilkins, Identifying and Avoiding Bias in Research, Plast. Reconstr. Surg. 126 (2010) 619-625.

[31] Innovation in Construction Equipment and Its Flow into the Construction Industry: Journal of Construction Engineering and Management: (ASCE), (n.d.).

[32] Caterpillar Inc., Caterpillar performance handbook, 42nd edition, (2012). 
[33] Komatsu Inc., Komatsu Specifications \& Application Handbook, 30th edition, (2009).

[34] P. Lewis, A. Hajji, Evaluation of Construction Equipment Fleets through Fuel Use and Emissions Inventories, in: Sustain. Transp. Syst., American Society of Civil Engineers, n.d.: pp. 138-145.

[35] Evaluating the Performance of Construction Equipment Operators in Egypt, J. Constr. Eng. Manag. 122 (1996) 109-114.

[36] S. Alwi, Factors influencing construction productivity in the Indonsesian context, in: Fac. Built Environ. Eng., Fukuoka, Japan, 2003. http://eprints.qut.edu.au/4154/ (accessed February 26, 2016).

[37] S. Dowdy, S. Wearden, D. Chilko, Statistics for research, 3rd ed., Wiley, 2004.

[38] H. Nabizadeh, H.F. Haghshenas, Y. Kim, F.T.S. Aragao, Effects of rejuvenators on high-RAP mixtures based on laboratory tests of asphalt concrete (AC) mixtures and fine aggregate matrix (FAM) mixtures, Construction and Building Materials 152 (2017) 65-73.

[39] H. Nabizadeh, Viscoelastic, Fatigue Damage, and Permanent Deformation Characterization of High Rap Bituminous Mixtures Using Fine Aggregate Matrix (FAM), (2015).

[40] H. Haghshenas, H. Nabizadeh, Y. Kim, K. Santosh, Research on high-rap asphalt mixtures with rejuvenators and WMA additives (2016).
[41] M Moosavi, H Nabizadeh, MH Mehranpour, A Nazem, A.N. Shirazi. An investigation of surface roughness measurement in rock joints with a 3D scanning device, (2013).

[42] H.N. Rafsanjani, Y Gholipoor, H. Nabizadeh, ESCMODEL: A Model for Optimum Machinery Selection in Earth-Fill Dam Projects, World Congress on Engineering and Computer Science 2010 (WCECS 2010)

[43] S. Amelian, M. Manian, S.M. Abtahi, A. Goli, Moisture sensitivity and mechanical performance assessment of warm mix asphalt containing by-product steel slag, Journal of Cleaner Production, 176 (2018) 329-337.

[44] S. Amelian, S.M. Abtahi, S.M. Hejazi, Moisture susceptibility evaluation of asphalt mixes based on image analysis, Construction and Building Materials, 63 (2014) 294-302.

[45] S. Amelian, Y. Kim, Evaluation of Tack Coating Practices for Asphalt Overlays in Nebraska. Nebraska Department of Transportation. SPR-P1(16) M039 (2017).

[46] H.N. Rafsanjani, M. Chehreghani, M. Nourbakhsh, Recycling of crushed clay brick as aggregate in concrete, Applied Mechanics and Materials 94 (2011) 1087-1090.

[47] M. Khezripour, H.N. Rafsanjani, Bucket with Flexible Width for Hoe, 2nd International Conference on Applied Research in Civil Engineering, Architecture, and Urban Planning (2015). 